\title{
Gerenciamento de resultados e eficiência: um estudo nas cooperativas de crédito filiadas ao sistema Unicred
}

\author{
Earnings management and efficiency: a study in credit unions affiliated to the Unicred system
}

\begin{abstract}
Resumo
Neste artigo propõe-se a verificar se as cooperativas incorrem na prática de gerenciamento de resultados com vistas a evitar divulgar perdas. Também, pretende-se analisar a relação entre eficiência e este tipo de gerenciamento de resultados nestas instituições a fim de compreender a adoção dessas práticas com finalidades estratégicas. Para tal, utilizou-se o teste qui-quadrado, numa amostra composta por 90 observações referentes às cooperativas ligadas à Confederação Nacional das Cooperativas Centrais Unicred's (UNICRED), no período de 2009 a 2014. Os resultados indicaram que há relação entre o gerenciamento de resultados para evitar divulgar perdas e os escores de eficiência nas cooperativas de crédito. A faixa de eficiência que mais gerenciou seus resultados a fim de evitar divulgar perdas foi a de eficiência na faixa mediana, podendo indicar que as cooperativas mais eficientes não necessitariam gerenciar seus resultados e que as cooperativas menos eficientes não teriam nenhum tipo de benefício ao adotar tal prática.
\end{abstract}

Palavras-chave: Gerenciamento de resultados; Eficiência; Cooperativas de crédito; Unicred

\begin{abstract}
In this paper we verify if credit unions incur in the practice of earnings management in order to avoid spreading losses. In addition, we analyze the relationship between efficiency and this type of earnings management in these institutions in order to understand the adoption of these practices for strategic purposes. For this, the chi-square test was used in a sample composed of 90 observations related to the cooperatives linked to the National Confederation of Unicred's Central Cooperatives (UNICRED), from 2009 to 2014. The results indicated that there is a relationship between the earnings management to avoid spreading losses and efficiency scores in credit unions. The efficiency range that most managed its results in order to avoid disclosing losses was that of efficiency in the middle range, which could indicate that the most efficient credit unions would not need to manage their results and that the less efficient credit unions would not have any kind of benefit when adopting such practice.
\end{abstract}

Keywords: Earnings management; Efficiency; Credit unions; Unicred

Recebido: 06/09/2017 Aceito: 16/10/2017

Lua Syrma Zaniah Santos ${ }^{1}$, Cecília Maria Andrade Guerra²

${ }^{1}$ Mestranda em Ciências Contábeis na UFMG - Lagoa Santa/MG - luasyrma@ gmail.com

2 Bacharel em Ciências Contábeis na UFMG - ceciliaandradeguerra@gmail.com 


\section{Introdução}

$\mathrm{A}$ s cooperativas de crédito são importantes instrumentos de inserção social e de desenvolvimento local. Estas organizações foram crescendo ao longo do tempo e ampliando suas áreas de atuação. Os dados consolidados apresentados no World Credit Union Conference, realizado no ano de 2015 nos EUA, demonstram a existência de, aproximadamente, $\quad 57.000$ cooperativas de crédito em todo o mundo, presentes em 105 países, totalizando cerca de 217 milhões de associados. Outro dado de destaque foi divulgado em um estudo realizado pelo Conselho Mundial das Cooperativas de Crédito, de que $8,2 \%$ da população mundial economicamente ativa está associada a uma cooperativa de crédito (WCUC, 2017). Como formas alternativas de acesso ao crédito e estando inseridas em um contexto de economias capitalistas e cada vez mais globalizadas, as cooperativas se tornam importantes opções para diversos setores da sociedade, desempenhando papel importante para a diminuição da pobreza e das desigualdades sociais, como observam Soares e Melo Sobrinho (2008).

Em diversos países, as Cooperativas de Crédito estão entre as principais instituições financeiras a serviço da população. Pode-se perceber na França, a maior expressão do cooperativismo de crédito do mundo, já que este país ocupa o primeiro lugar no ranking dos países com maior expressão no cooperativismo financeiro, tendo movimentado no ano de 2013 cerca de US\$ 2 trilhões em empréstimos. (PORTAL DO COOPERATIVISMO FINANCEIRO, 2017). A partir do Portal do Cooperativismo Financeiro (2017), infere-se que no mês de dezembro de 2016, o Brasil contava com 1.000 cooperativas de crédito, sendo que destas, $75 \%$ possuíam ligação com algum dentre os sistemas cooperativistas: Sistema de Cooperativas de Crédito do Brasil (SICOOB), Sistema de Crédito Cooperativo (SICREDI), Confederação Nacional das Cooperativas Centrais (UNICRED), Sistema Cooperativo de Crédito Urbano (CECRED), Sistema das Cooperativas de Crédito Rural com Interação Solidária (CRESOL) e Uniprime. Estas instituições detêm aproximadamente $90 \%$ da rede de atendimento e do total de associados. De acordo com informações constantes no Portal (2017), dados do Banco Central acerca das informações consolidadas destas organizações retratam que no mês de dezembro de 2016, as Cooperativas Financeiras no Brasil administravam ativos totais de R\$ 296 bilhões, o que correspondeu a 3,5\% do total de ativos das instituições financeiras do país. E em 2016, as cooperativas de crédito correspondiam a $6^{\mathrm{a}}$ instituição financeira do país no quesito de ativos totais e $5^{\mathrm{a}}$ em patrimônio líquido (PORT, 2017).

Dentre os principais sistemas cooperativistas do Brasil, tem-se a Confederação Nacional das Cooperativas Centrais Unicred's - Unicred do Brasil, que é o foco da análise neste estudo. O sistema Unicred foi criado em 1989, no Rio Grande do Sul, suprindo uma necessidade de se ter uma instituição que representasse os interesses de suas cooperativas centrais e singulares. Atualmente, as cooperativas do sistema Unicred atuam em dez estados do país com aproximadamente 230 unidades de negócios, por meio de quatro centrais e 181.906 cooperados, estando entre os maiores sistemas cooperativistas do Brasil (UNICRED, 2017).

Apesar de alguns aspectos peculiares relativos ao funcionamento das cooperativas de crédito, estas são instituições financeiras e desempenham um papel singular na economia. Desta forma, a manutenção de suas operações e a continuidade de suas atividades, são de grande importância para o Sistema Financeiro Nacional e a sua solidez. $\mathrm{O}$ bom funcionamento das instituições financeiras está ligado ao crescimento econômico de um país, sendo por isso importante uma investigação acerca da eficiência nestas instituições.

As cooperativas de crédito são uma alternativa de acesso aos serviços financeiros com inúmeros benefícios, tal como maior rapidez e flexibilidade nas operações, mantidas pelos próprios cooperados a fim de conseguirem uma melhor administração dos seus recursos financeiros. Além disso, são consideradas "eficientes para o fortalecimento da economia, a democratização do crédito e a desconcentração de renda" (ARAÚJO, 2011; PORTAL DO COOPERATIVISMO DE CRÉDITO, 2015).

A Lei $\mathrm{n}^{\circ}$ 5.764/71, juntamente com a NBC T 10.8 e NBC T 10.21, estabelecem a destinação do resultado, das sobras e das perdas nas sociedades cooperativas. Assim, as sobras líquidas serão distribuídas conforme a deliberação da Assembleia Geral, aos associados. Esta será realizada, proporcionalmente à produção entregue pelos cooperados, assim como às operações realizadas 
pelos mesmos. Em relação às perdas do exercício, se não forem totalmente absorvidas pela Reserva Legal, estas poderão ser rateadas entre os associados, uma vez que não deverá haver saldo acumulado de exercício anterior. O conjunto de estudos e de normas peculiares destas organizações, podem levar às pressões por melhora do desempenho por parte dos associados. Para isso, tais organizações esperam demonstrar cada vez mais eficiência ao mercado financeiro, e para chegar a esse patamar ou apresentarem melhores resultados que outras instituições, muitas delas podem se valer do gerenciamento de resultados. Portanto, deve-se verificar se as cooperativas de crédito que gerenciam seus resultados para evitar divulgar perdas levam a um maior escore de eficiência, a um menor escore, ou se esses aspectos acabam não tendo nenhuma relação de impacto um sobre o outro.

Paralelamente, diversos estudos têm sido realizados acerca do tema de gerenciamento de resultados. No Brasil, Martinez (2001) foi um autor precursor sobre a temática no âmbito nacional. A partir de então, Perlingueiro (2009) evidenciou que a maioria das produções científicas no país, até então, era voltada para as companhias abertas. O mercado de capitais também obteve bastante ênfase nas pesquisas, conforme observa Maia et al. (2013). Assim, estudos nas instituições financeiras, tais como os de Fuji e Carvalho (2005), Goulart (2007) e Dantas et al. (2013), ainda constam em menor proporção em relação aos demais temas abordados. Especificamente, no que tange às cooperativas, os estudos são ainda incipientes. O Sistema das Cooperativas de Crédito no Brasil (SICOOB) foi avaliado por Maia et al. (2013), bem como Silva Júnior \& Bressan (2014) e Bressan et al. (2015b) avaliaram a prática da manobra contábil para as cooperativas do Sistema de Crédito Cooperativo (SICREDI). O sistema Confesol, atualmente denominado Confederação Cresol também foi objeto de estudo por Bressan et al. (2015a).

Desta forma, este estudo pretende contribuir com a expansão das análises acerca dos sistemas cooperativistas brasileiros, auxiliando para uma análise geral destas cooperativas. Além disto, suprir lacunas sobre estudos específicos sobre este tipo de instituição, bem como subsidiar e elaborar análises úteis para órgãos regulatórios, como o Banco Central do Brasil, a fim de descrever o comportamento destas instituições, tão importantes no contexto do sistema financeiro nacional.

Segundo Cuevas e Fischer (2006), as cooperativas de crédito estão entre as instituições financeiras menos estudadas, sendo que estas requerem maiores pesquisas acerca das suas particularidades. Portanto, esta pesquisa justifica-se pela importância do tema no âmbito das instituições financeiras, além de que "a adoção de mecanismos de controles gerenciais e de avaliação de desempenho ainda é pouco utilizada para o desenvolvimento das cooperativas" (VILELA; NAGANO; MERLO, 2007). O presente trabalho pode contribuir ainda para a verificação da relação do gerenciamento de resultados a fim de evitar divulgar perdas nas cooperativas de crédito, com os escores de eficiência apresentados por elas, ou seja, uma abordagem inovadora na produção científica da área.

Estudos empíricos investigaram a prática de gerenciamento de resultados em instituições financeiras, em suas diversas modalidades, tais como Fuji e Carvalho (2005), Zendersky (2005), Goulart (2007) e Dantas et al. (2013). Em todos estes trabalhos, confirmou-se que haviam indícios de ocorrência de tais práticas nas instituições estudadas.

Em relação aos principais sistemas cooperativistas brasileiros, foram estudados o Sicoob, o Sicredi, a Confesol e a Unicred, a respeito do tema de gerenciamento de resultados. Todos estes estudos apontaram para a ocorrência desta prática contábil. No caso do Sicoob, Maia et al. (2013) pesquisaram três modalidades de gerenciamento, sendo duas delas confirmadas: o tipo de gerenciamento para evitar divulgar perdas e o gerenciamento que busca a suavização dos resultados. O Sistema de Crédito Cooperativo (Sicred) foi estudado por Silva Júnior e Bressan (2014) e também por Bressan et al. (2015b), em relação aos gerenciamentos de resultados do tipo income smoothing e do tipo para evitar a divulgação de perdas, respectivamente. Em ambos os casos, os resultados confirmaram a realização do gerenciamento. O terceiro sistema investigado foi a Confesol, acerca do gerenciamento de resultados com vistas a não divulgar perdas, feito por Bressan et al. (2015a). Por último, Santos (2015) investigou se haviam indícios de gerenciamento de resultados com vistas a evitar a divulgação de perdas nas cooperativas ligadas à Unicred. Todos estes estudos 
contribuem para a pesquisa acerca do comportamento das cooperativas de crédito brasileiras, em relação à prática do gerenciamento de resultados.

Neste contexto, esta pesquisa propõe-se a verificar se as cooperativas ligadas à Confederação Nacional das Cooperativas Centrais Unicred's (UNICRED), incorreram na prática de gerenciamento de resultados com vistas a evitar divulgar perdas, no período compreendido entre os anos de 2009 a 2014, bem como analisar a relação entre eficiência e gerenciamento de resultados para evitar divulgar perdas nestas instituições, no mesmo período, a fim de compreender a adoção dessas práticas com finalidades estratégicas dentro dessas entidades.

\section{Revisão da literatura e estudos anteriores}

\subsection{Cooperativismo de Crédito}

Para dar suporte a este estudo sobre a ocorrência de gerenciamento de resultados nas cooperativas de crédito, inicialmente é necessário entender sobre alguns aspectos relevantes sobre estas instituições. As cooperativas de crédito têm sua origem datada no ano de 1844, no bairro de Rochdale-Manchester, na Inglaterra, quando surge a primeira cooperativa moderna: a Sociedade dos Probos de Rochdale. Esta Sociedade foi formada a partir da junção de vinte e oito tecelões, que buscavam por uma alternativa à exploração que sofriam sob o sistema capitalista, no contexto da Revolução Industrial. O êxito desta Sociedade abriu caminho para a formação de diversas outras cooperativas. A partir de então, formou-se a base para os princípios cooperativistas, que foram sendo aprimorados e funcionam como fundamentos norteadores para estas instituições. (PORTAL DO COOPERATIVISMO FINANCEIRO, 2017).

Conforme a Organização das Cooperativas Brasileiras (OCB, 2017), os princípios cooperativistas são: Adesão voluntária e livre, Gestão democrática, Participação econômica dos membros, Autonomia e independência, Educação, formação e informação, Intercooperação e o Interesse pela comunidade.

No Brasil, a experiência com o cooperativismo de crédito é historicamente retratada no ano de 1902, quando o padre suíço Theodor Amstad iniciou e fundou a Cooperativa Caixa de Economia e Empréstimos de Nova Petrópolis, que se transformou na Cooperativa de Crédito de Livre Admissão de Associados Pioneira da Serra Gaúcha Sicredi Pioneira RS. (SOARES E MELO SOBRINHO, 2008).

Estas instituições representam o meio pelo qual determinado grupo de pessoas possa atingir seus objetivos através de um acordo voluntário para cooperação recíproca (ANCOSOL, 2015). Para Silva e Bacha (2007), as cooperativas de crédito são instituições sem fins lucrativos, de ajuda mútua, pertencentes ao sistema financeiro nacional, que devem ser de propriedade e controle dos sócios e que têm por finalidade a prestação de serviços de crédito aos seus associados.

Atualmente, além da legislação básica que rege o Sistema Financeiro Nacional de forma abrangente e a própria Constituição Federal, o cooperativismo de crédito está regulamentado de acordo com a Lei 4.595/64 , que instituiu a Reforma Bancária em 1964; a Lei 5.764/71, que consiste na Lei do Cooperativismo Brasileiro, a Lei Complementar 130/2009 e a Resolução 4.434/2015, do Conselho Monetário Nacional (PORTAL DO COOPERATIVISMO FINANCEIRO, 2017).

A Lei $\mathrm{n}^{\circ}$ 5.764/1971 dispõe sobre as categorias das cooperativas, segundo a qual podem ser singulares, centrais ou federações, bem como confederações de cooperativas. No Brasil, o cooperativismo de crédito está organizado em sistemas cooperativos, que geralmente envolvem cooperativas de todos os níveis. A referida Lei ressalta ainda, que "as cooperativas são sociedades de pessoas, com forma e natureza jurídica próprias, de natureza civil, não sujeitas a falência, constituídas para prestar serviços aos associados".

\subsection{Gerenciamento de resultados}

Segundo Rodrigues et al. (2007), no Brasil, o gerenciamento de resultados é uma expressão que se refere a um conjunto de práticas adotadas por gestores e contadores com o intuito de obter os resultados contábeis desejados, dentro dos limites legais contábeis. Complementarmente, Grecco (2013) destaca que o gerenciamento de resultados configura uma espécie de gestão intencional dos resultados de uma empresa, que pode ocorrer através do uso de técnicas e julgamentos contábeis, não se confundindo com a fraude, uma vez que este ocorre 
dentro dos limites legais da norma e da prática contábil.

De forma ainda mais ampla, Martinez (2013, p.5) apresenta os itens que caracterizam a prática do Gerenciamento de Resultados:

Uso da discricionariedade gerencial na realização de escolhas contábeis (reconhecimento e mensuração), na tomada de decisões operacionais e na seleção dos critérios de apresentação da demonstração de resultado (disclosure). Todas dentro dos limites das normas contábeis, visando influenciar as percepções sobre fatos econômicos subjacentes, que são refletidos nas medidas de resultado contábil.

No que tange às técnicas, Martinez (2001) destaca três tipos de gerenciamento: o target earnings que é o gerenciamento para alterar os resultados contábeis na intenção de alcançar metas estabelecidas; o income smoothing, que visa a redução da oscilação de resultados; e o take bath accouting, que tem por finalidade reduzir os resultados contábeis, com o intuito de aumentar os resultados futuros. Além disso, o mesmo autor classifica o gerenciamento em categorias, a saber: Gerenciamento por accruals (GRA); e por decisões operacionais (GRDO). Entre suas diferenças fundamentais, está o impacto no fluxo de caixa operacional. As decisões operacionais têm impacto direto no fluxo de caixa da empresa, enquanto os accruals não têm necessariamente essa relação. $\mathrm{O}$ presente trabalho trata do gerenciamento para evitar divulgar perdas, que é um gerenciamento referente às decisões operacionais do tipo target earnings.

Martinez (2001), Goulart (2007), Maia et al. (2013), Reis (2015) e Bressan, et al. (2015a) investigaram o gerenciamento de resultados com vistas a evitar divulgar perdas.

Martinez (2001) buscou evidenciar empiricamente que as empresas de capital aberto brasileiras gerenciavam seus resultados em consequência aos estímulos do mercado de capitais e confirmou as evidências de que estas empresas evitaram divulgar perdas anuais e trimestrais para o período compreendido dos anos de 1995 a 1999. Goulart (2007) avaliou se as 50 maiores instituições financeiras brasileiras se utilizaram da contabilização de suas operações de crédito, títulos de valores mobiliários e derivativos, para gerenciar os resultados contábeis entre os anos de 2002 a 2006.
No tocante às cooperativas de crédito, ainda há muito que se explorar na investigação a respeito do gerenciamento de resultados. Maia et al. (2013) representa um marco na pesquisa nacional. Esses autores verificaram a ocorrência de gerenciamento em três modalidades: para evitar divulgar perdas, para suavização de resultados e também para adequação de capital mínimo, situação tratada pelo acordo de Basiléia. $\mathrm{O}$ estudo avaliou as cooperativas vinculadas ao Sistema das Cooperativas de Crédito do Brasil (SICOOB), no período entre 2001 e 2011, e concluiu que nas modalidades de evitar reportar perdas e suavizar seus resultados, as cooperativas do Sicoob incorreram na prática de gerenciamento. Isso não se confirmou no gerenciamento para adequação do capital regulatório nestas instituições.

Silva Junior e Bressan (2014) testaram a presença do gerenciamento de resultados do tipo income smoothing nas cooperativas filiadas ao sistema Sicredi, no mesmo período de análise pesquisado por Maia et al. (2013). Para tal, se utilizaram de uma regressão múltipla com dados em painel e inferiram que as cooperativas do Sistema de Crédito Cooperativo (Sicredi), também gerenciaram seus resultados na modalidade analisada. O mesmo sistema cooperativo foi estudado, tomando-se como base os anos de 2000 a 2010, desta vez na modalidade de gerenciamento para não divulgar perdas, no trabalho de Bressan et al. (2015a). As evidências indicaram que as cooperativas analisadas praticaram o gerenciamento de resultados, tanto nos resultados semestrais, como especialmente nos anuais.

\subsection{Eficiência}

Derivado do latim efficientia, que significa virtude ou força para se produzir um determinado resultado, a eficiência técnica pode ser chamada de eficiência produtiva ou medida de produtividade global.

Ferreira, Gonçalves e Braga (2007 p.428) esclarecem que "nas cooperativas de crédito, a eficiência está associada à capacidade de maximização dos benefícios aos cooperados, materializados em operações de créditos e benefícios líquidos, em contrapartida aos recursos empregados para sua obtenção. Os autores descrevem que as medidas de eficiência são normalmente representadas por uma função de fronteiras 
construídas no sistema das coordenadas, em que as empresas eficientes se encontram sobre a fronteira. Com a existência de desperdícios, nem todas as firmas sobre a fronteira são realmente eficientes, porém diante das suas restrições, essas empresas conseguem produzir o máximo possível. Assim, para identificar uma empresa ineficiente bastaria verificar a distância em que a mesma se encontra abaixo da fronteira de produção.

Berger e Humphrey (1997) verificaram cinco tipos de abordagens para determinação da fronteira eficiente. Sendo que três delas utilizam métodos paramétricos: a stochastic frontier approach (SFA), a distribution free approach (DFA) e a thick frontier approach (TFA); e duas delas utilizam métodos não paramétricos: o data envelopment analysis (DEA) e a free disposal hull (FDH). O modelo mais utilizado segundo Tabak, Krause e Portella (2005) é o DEA. A fronteira DEA é formada pela combinação linear dos pontos que conectam o conjunto das melhores práticas, formando um conjunto convexo de possibilidades de produção. A diferença entre a DEA e as abordagens paramétricas é que ela não é determinada por uma forma funcional específica, mas sim gerada a partir dos dados das instituições analisadas, o que evita a possibilidade de equívoco de especificação da forma funcional da fronteira (BERGER E HUMPHREY, 1997).

Segundo Tabak, Fazio e Cajueiro (2011), as melhorias em termos de eficiência surgem da possibilidade de concentração da carteira de crédito nos bancos, por meio da especialização nos serviços. Com isso, surge a melhora no desempenho dessas entidades, tanto em termos de retorno quanto na diminuição da inadimplência.

Vilela, Nagano e Merlo (2007) mostraram em seu estudo que no estado de São Paulo, o volume de operações de crédito das cooperativas diminuiu, nos anos de 2001 e 2002, apontando que estas estão operando com ineficiência operacional. Resultados semelhantes também foram encontrados por Bressan et al. (2010) nas cooperativas de crédito do estado de Minas Gerais, e por Dambros, Lima e Figueiredo (2009) nas cooperativas de crédito no estado do Paraná.

\section{Procedimentos metodológicos}

\subsection{Amostra e fonte de dados}

Para a elaboração das análises, utilizou-se como base as investigações de Santos (2015), de Bittencourt (2015) e de Guerra (2016) que trabalharam os dados a partir das informações públicas disponíveis no site do Banco Central do Brasil e disponibilizaram as bases de dados para execução desta pesquisa. Desta forma, utilizando-se dos achados destes estudos, foi possível analisar se existe relação entre o gerenciamento de resultados para evitar divulgar perdas e os escores de eficiência nas cooperativas de crédito ligadas à Confederação Nacional das Cooperativas Centrais Unicred's (UNICRED) no período de 2009 a 2014. Santos (2015) avaliou se as cooperativas ligadas à UNICRED realizaram gerenciamento de seus resultados com vistas a evitar divulgar perdas, no período de 2005 a 2014. Para tal, a análise de frequência em histogramas, proposta por Burgstahler e Dichev (1997) foi utilizada, para verificar a possível ocorrência deste tipo de gerenciamento de resultados neste grupo de cooperativas. No presente estudo, foi considerada para análise a amostra referente às cooperativas de crédito filiadas à UNICRED que se encontram em ambos os trabalhos descritos acima, para que assim pudesse ser realizada análise da eficiência e do gerenciamento de resultados para evitar divulgar perdas em cada cooperativa. Portanto, a amostra foi composta por 15 cooperativas de crédito vinculadas ao sistema Unicred com dados referentes a 2009 a 2014, sendo este o período de dados mais atual, quando da coleta de dados para realização desta pesquisa, totalizando 90 observações.

\subsection{Modelo analítico}

Para confrontar se as cooperativas de crédito filiadas à Unicred que possuíam altos escores de eficiência conforme resultados de Bittencourt (2015) gerenciaram ou não seus resultados para evitar divulgar perdas no período de 2009 a 2014 foi utilizado o modelo de teste $\chi^{2}$ sendo este um teste não paramétrico utilizado na comparação entre duas ou mais amostras, em que a sua estatística é igual a diferença do quadrado entre as frequências observadas e esperadas, dividida pela frequência esperada em cada célula, somada ao longo de todas as células, conforme (1) (ANDERSON et al., 2003). 
Fórmula: $\chi^{2}=\sum \frac{\left(f_{o}-f_{e}\right)^{2}}{f_{e}}$

Em que:

$f_{o}=$ frequência observada

$f_{e}=$ frequência esperada

$f_{e}=$ (soma das linhas $\mathrm{x}$ soma das colunas) / $\mathrm{n}$, em que:

Soma das linhas = soma de todas as frequências na linha

Soma das colunas $=$ soma de todas as frequências na coluna

$\mathrm{n}=$ tamanho da amostra

Para Anderson et al. (2013), este teste trabalha com informações qualitativas e com ele é possível realizar comparações entre frequências em que caso as diferenças entre as frequências observadas e as esperadas em cada categoria forem muito pequenas ou próximas de zero, os dois grupos se portam de maneira semelhante.

Após a coleta e organização dos dados, iniciaram-se as realizações dos testes estatísticos através do auxílio da ferramenta da planilha eletrônica Excel para avaliar se existe ou não relação entre o gerenciamento de resultados para evitar perdas e os escores de eficiência das cooperativas de crédito filiadas à Unicred, conforme hipótese nula do teste $\chi^{2}$, de que não existe relação entre $o$ gerenciamento de resultados para evitar divulgar perdas e os escores de eficiência das cooperativas de crédito filiadas à Unicred.
As observações das cooperativas de crédito associadas ao sistema Unicred foram segregadas em cooperativas que gerenciaram seus resultados para evitar divulgar perdas nos períodos analisados e as cooperativas que não gerenciaram. Por outro lado, foram separadas as cooperativas que alcançaram $100 \%$ de eficiência das demais cooperativas que não alcançaram o escore máximo. Estas foram segregadas em faixas de $20 \%$ de modo a realizar uma análise mais específica por faixas de eficiência.

A partir da matriz dos dados observados e da matriz dos dados esperados, gera-se o valor-p para o teste $\chi^{2}$ a fim de comparar com o nível de significância (alfa $-\alpha$ ), e a partir dessa análise identificar se a hipótese nula deve ou não ser rejeitada (ANDERSON et al., 2003). Para esse trabalho, foi estipulado o intervalo de confiança de 99\%, sendo este considerado um bom parâmetro de confiança dos dados a serem analisados.

\section{Análise dos resultados}

A partir dos dados dos trabalhos bases (SANTOS, 2015; BITTENCOURT, 2015 e GUERRA, 2016), foi possível elencar as cooperativas de crédito filiadas à Unicred que possuem tanto as informações de eficiência, quanto de gerenciamento de resultados durante o período analisado, conforme exposta na Tabela 1. A partir destes dados foi realizado o teste de $\chi^{2}$ para verificar a relação entre essas variáveis.

Tabela 1 - Escores de eficiência e gerenciamento de resultados nas cooperativas analisadas

\begin{tabular}{c|c|c|c|c|c|c|c|c|c|c|c|c}
\hline \multirow{2}{*}{$\begin{array}{c}\text { Coope- } \\
\text { rativa }\end{array}$} & \multicolumn{2}{|c|}{$\mathbf{2 0 0 9}$} & \multicolumn{2}{c|}{$\mathbf{2 0 1 0}$} & \multicolumn{2}{c|}{$\mathbf{2 0 1 1}$} & \multicolumn{2}{c|}{$\mathbf{2 0 1 2}$} & \multicolumn{2}{c|}{$\mathbf{2 0 1 3}$} & \multicolumn{2}{c}{$\mathbf{2 0 1 4}$} \\
\hline & $\begin{array}{c}\text { Efici- } \\
\text { ência }\end{array}$ & GR & $\begin{array}{c}\text { Efici- } \\
\text { encia }\end{array}$ & GR & $\begin{array}{c}\text { Efici- } \\
\text { ência }\end{array}$ & GR & $\begin{array}{c}\text { Efici- } \\
\text { ência }\end{array}$ & GR & $\begin{array}{c}\text { Efici- } \\
\text { encia }\end{array}$ & GR & $\begin{array}{c}\text { Efici- } \\
\text { ência }\end{array}$ & GR \\
\hline C 1 & $94,28 \%$ & 0 & $89,83 \%$ & 0 & $100,00 \%$ & 0 & $64,76 \%$ & 1 & $65,97 \%$ & 1 & $64,62 \%$ & 0 \\
\hline C 2 & $40,56 \%$ & 0 & $47,03 \%$ & 0 & $41,97 \%$ & 0 & $43,30 \%$ & 1 & $54,73 \%$ & 1 & $47,87 \%$ & 1 \\
\hline C 3 & $100,00 \%$ & 0 & $77,33 \%$ & 1 & $69,72 \%$ & 1 & $61,82 \%$ & 1 & $66,45 \%$ & 1 & $60,97 \%$ & 0 \\
\hline C 4 & $33,70 \%$ & 0 & $31,51 \%$ & 0 & $38,19 \%$ & 0 & $39,11 \%$ & 0 & $41,54 \%$ & 0 & $100,00 \%$ & 0 \\
\hline C 5 & $58,06 \%$ & 1 & $50,32 \%$ & 1 & $65,26 \%$ & 0 & $78,27 \%$ & 0 & $66,14 \%$ & 0 & $72,95 \%$ & 1 \\
\hline C 6 & $48,32 \%$ & 0 & $41,22 \%$ & 1 & $31,44 \%$ & 0 & $96,01 \%$ & 0 & $32,56 \%$ & 0 & $100,00 \%$ & 0 \\
\hline C 7 & $37,78 \%$ & 1 & $42,44 \%$ & 0 & $45,54 \%$ & 0 & $50,21 \%$ & 0 & $56,47 \%$ & 0 & $58,10 \%$ & 0 \\
\hline C 8 & $100,00 \%$ & 0 & $31,76 \%$ & 1 & $71,17 \%$ & 0 & $78,26 \%$ & 1 & $71,27 \%$ & 1 & $70,82 \%$ & 0 \\
\hline C 9 & $57,57 \%$ & 0 & $59,52 \%$ & 0 & $62,07 \%$ & 1 & $63,72 \%$ & 0 & $66,47 \%$ & 0 & $63,77 \%$ & 1 \\
\hline
\end{tabular}




\begin{tabular}{c|c|c|c|c|c|c|c|c|c|c|c|c} 
& & & & & & & & & & & & \\
\hline C 10 & $48,90 \%$ & 0 & $38,06 \%$ & 1 & $39,37 \%$ & 1 & $48,39 \%$ & 1 & $85,11 \%$ & 0 & $60,93 \%$ & 1 \\
\hline C 11 & $68,63 \%$ & 0 & $71,26 \%$ & 0 & $86,07 \%$ & 0 & $98,27 \%$ & 0 & $100,00 \%$ & 0 & $100,00 \%$ & 0 \\
\hline C 12 & $77,88 \%$ & 1 & $95,00 \%$ & 1 & $100,00 \%$ & 0 & $69,70 \%$ & 1 & $53,74 \%$ & 0 & $88,10 \%$ & 1 \\
\hline C 13 & $100,00 \%$ & 0 & $100,00 \%$ & 0 & $100,00 \%$ & 0 & $90,16 \%$ & 0 & $84,62 \%$ & 0 & $66,38 \%$ & 1 \\
\hline C 14 & $100,00 \%$ & 1 & $89,03 \%$ & 0 & $100,00 \%$ & 0 & $73,15 \%$ & 1 & $77,93 \%$ & 1 & $74,48 \%$ & 0 \\
\hline C 15 & $56,98 \%$ & 1 & $63,99 \%$ & 1 & $61,11 \%$ & 1 & $61,85 \%$ & 1 & $73,34 \%$ & 0 & $67,38 \%$ & 0 \\
\hline
\end{tabular}

Fonte: Resultados da pesquisa a partir dos dados de Santos (2015) e Bittencourt (2015).

GR = 0 quando não foi detectado indícios de gerenciamento de resultados para evitar divulgar perdas, e GR = 1 quando foi detectado indícios de gerenciamento de resultados para evitar divulgar perdas.

Das quinze cooperativas presentes na amostra, seis estão localizadas no estado de Santa Catarina, quatro em São Paulo, duas no Rio Grande do Sul, uma no Rio de Janeiro, uma em Minas Gerais e uma no Mato Grosso.

A partir da disposição dos dados, é possível verificar cooperativas com informações parecidas ao longo dos anos analisados, às quais pode-se analisar de forma a obter a caracterização sobre a amostra em questão. As cooperativas C 3 e C 15 gerenciaram seus resultados por quatro anos consecutivos dentro do período analisado, sendo que ambas estão presentes no Sul do país; a C 3 em Santa Catarina; a C 15 está presente no Rio Grande do Sul. As cooperativas de crédito $\mathrm{C} 4$ e $\mathrm{C} 11$ foram as únicas cooperativas que não gerenciaram seus resultados para evitar divulgar perdas durante o período analisado. A C 4 pertence ao tipo de cooperativa de Crédito Mútuo referente à Atividade Profissional. A $\mathrm{C} 11$ pertence ao mesmo tipo de cooperativa que a $\mathrm{C}$ 4 e está situada no estado de São Paulo. (BANCO CENTRAL DO BRASIL, 2016a; BANCO CENTRAL DO BRASIL, 2016b).
A C 13 foi a mais eficiente da amostra, com escores de eficiência de $100 \%$ durante três anos consecutivos. Esta cooperativa, fundada em $1993 \mathrm{em}$ Florianópolis/Santa Catarina. Além da C 13, as cooperativas de crédito com maiores médias de eficiência durante o período foram as C 11, C 12 e C 14. A C 11 foi uma das cooperativas que não gerenciou seus resultados durante o período conforme já explicitado. As cooperativas C 12 e C 14 gerenciaram seus resultados durante quatro e três anos do período analisado, respectivamente. Ambas cooperativas são singulares e estão localizadas no estado de Santa Catarina. (UNICRED FLORIANÓPOLIS, 2016; BANCO CENTRAL DO BRASIL, 2016a; BANCO CENTRAL DO BRASIL, 2016b).

As cooperativas de crédito que apresentaram menores médias de eficiência, durante o período analisado, foram C 2, C 4 e C 7. A C 4 foi uma que não gerenciou seus resultados para evitar divulgar perdas durante o período. As C 2 e C 7 localizam-se no estado de São Paulo.

A partir dos dados, obteve-se as matrizes (Tabela 2) de dados observados e esperados tendo como base a eficiência e o gerenciamento de resultados para evitar divulgar perdas, além do resultado do teste qui-quadrado: 
Tabela 2 - Matrizes de Dados Observados e Esperados e Resultado Teste Qui-Quadrado

\begin{tabular}{|c|c|c|c|c|c|c|}
\hline \multicolumn{7}{|c|}{ OBSERVADO } \\
\hline \multirow{2}{*}{ Coope rativas que } & \multicolumn{6}{|c|}{ Escores de Eficiência } \\
\hline & $20 \%<40 \%$ & $40 \%<60 \%$ & $60 \%<80 \%$ & $80 \%<100 \%$ & $100 \%$ & Total \\
\hline Gerenciam resultados & 4 & 8 & 20 & \begin{tabular}{|l|}
2 \\
\end{tabular} & 1 & 35 \\
\hline Não gerenciam resultados & 6 & 14 & 14 & 9 & 12 & 55 \\
\hline Total & 10 & 22 & 34 & 11 & 13 & 90 \\
\hline \multicolumn{7}{|c|}{ ESPERADO } \\
\hline \multirow{2}{*}{ Cooperativas que } & \multicolumn{6}{|c|}{ Escores de Eficiência } \\
\hline & $20 \%<40 \%$ & $40 \%<60 \%$ & $60 \%<80 \%$ & $80 \%<100 \%$ & $100 \%$ & Total \\
\hline Gerenciam resultados & 3,89 & 8,56 & 13,22 & \begin{tabular}{|l|}
4,28 \\
\end{tabular} & 5,06 & 35 \\
\hline Não gerenciam resultados & 6,11 & 13,44 & 20,78 & 6,72 & 7,94 & 55 \\
\hline Total & 10 & 22 & 34 & 11 & 13 & 90 \\
\hline Valor - p & \multicolumn{6}{|c|}{0,010996748} \\
\hline \multicolumn{7}{|c|}{ OBSERVADO } \\
\hline \multirow{2}{*}{ Coope rativas que } & \multicolumn{6}{|c|}{$\begin{array}{c}\text { Escores de Eficiência } \\
\end{array}$} \\
\hline & $20 \%<40 \%$ & $40 \%<60 \%$ & $60 \%<80 \%$ & $80 \%<100 \%$ & $100 \%$ & Total \\
\hline Gerenciam resultados & 4 & 8 & 20 & \begin{tabular}{|l|}
2 \\
\end{tabular} & 1 & 35 \\
\hline Não gerenciam resultados & 6 & 14 & 14 & 9 & 12 & 55 \\
\hline Total & 10 & 22 & 34 & 11 & 13 & 90 \\
\hline \multicolumn{7}{|c|}{ ESPERADO } \\
\hline \multirow{2}{*}{ Cooperativas que } & \multicolumn{6}{|c|}{$\begin{array}{c}\text { Escores de Eficiência } \\
\end{array}$} \\
\hline & $20 \%<40 \%$ & $40 \%<60 \%$ & $60 \%<80 \%$ & $80 \%<100 \%$ & $100 \%$ & Total \\
\hline Gerenciam resultados & 3,89 & 8,56 & 13,22 & \begin{tabular}{|l|}
4,28 \\
\end{tabular} & 5,06 & 35 \\
\hline Não gerenciam resultados & 6,11 & 13,44 & 20,78 & 6,72 & 7,94 & 55 \\
\hline Total & 10 & 22 & 34 & 11 & 13 & 90 \\
\hline Valor - p & & & 0,0109 & 996748 & & \\
\hline
\end{tabular}

Fonte: Resultados da Pesquisa

Com base na disposição desses dados é possível verificar que das 90 observações, em 35 (39\%), as cooperativas gerenciaram seus resultados para evitar divulgar perdas. Contudo, do total de cooperativas que gerenciaram seus resultados, 23 cooperativas, que representaram $(66 \%)$ chegaram a pelo menos $60 \%$ de eficiência. Já das cooperativas que não gerenciaram seus resultados, aproximadamente, $62 \%$ apresentaram escore de eficiência menor que $80 \%$, o que corresponde a 34 cooperativas. Assim, vê-se necessário aplicar um teste estatístico para avaliar de maneira formal a relação entre essas variáveis.

O teste qui-quadrado realizado, considerando o intervalo de confiança de $99 \%$, permitiu rejeitar a hipótese nula. Portanto, existe relação entre o gerenciamento de resultados para evitar divulgar perdas e os escores de eficiência das cooperativas de crédito filiadas à Unicred durante $\mathrm{o}$ período analisado.
É importante ressaltar também que das treze observações que alcançaram $100 \%$ de eficiência durante o período analisado, apenas uma delas gerenciou seus resultados a fim de evitar divulgar perdas, demonstrando pouca motivação para gerenciamento de resultados nas instituições mais eficientes do grupo. As cooperativas que estão na fronteira de eficiência não necessitam gerenciar seus resultados para chegarem a esse patamar. Essas treze observações são compostas por nove cooperativas de crédito, sendo elas as C 1, C 3, C 4, C 6, C 8, C 11, C 12, C 13 e C 14. Dentre estas, seis delas alcançaram $100 \%$ de eficiência em apenas um ano dentro do período analisado, duas conseguiram chegar a $100 \%$ em dois anos, e apenas uma alcançou o mais alto nível de eficiência durante três anos. Das cooperativas de crédito que alcançaram $100 \%$ de eficiência, seis delas se encontram em Santa Catarina, duas em São Paulo e uma no Rio de Janeiro, além de que oito delas são do tipo de 
cooperativa de crédito mútuo e uma delas do tipo de cooperativa de livre admissão.

Dentro da faixa de eficiência de $80 \%$ a $100 \%$, a proporção entre as cooperativas de crédito que gerenciaram e as que não gerenciaram seus resultados para evitar divulgar perdas está em linha com a proporção das cooperativas de crédito que alcançaram $100 \%$ de eficiência. Logo, as cooperativas de crédito com alta eficiência não têm a necessidade de gerenciar seus resultados. As onze observações nesta faixa referem-se a sete cooperativas de crédito da amostra.

Nos grupos de baixa eficiência, localizadas nas faixas de $20 \%$ a $40 \%$ e $40 \%$ a $60 \%$ e composta de 32 observações, a maioria, sendo esta composta por vinte observações, não gerenciou seus resultados. O total de observações nestas faixas referem-se a dez cooperativas de crédito da amostra, em que duas alcançaram no máximo $60 \%$ de eficiência durante todos os anos analisados e três cooperativas estiveram nessas faixas em apenas um ano do período analisado.

A faixa de eficiência de $60 \%$ a $80 \%$ apresentou resultado diferente das demais faixas, em que das trinta e quatro observações da amostra, vinte gerenciaram seus resultados para evitar divulgar perdas, sendo assim um percentual de $66 \%$ das observações desse grupo. Essa faixa é composta por onze cooperativas de crédito sendo elas as $\mathrm{C} 1, \mathrm{C} 3$, C 5, C 8, C 9, C 10, C 11, C 12, C 13, C 14 e C 15; em que dentro dos seis anos analisados, duas delas apresentaram observações dentro dessa faixa de eficiência em cinco anos consecutivos; e três delas apresentaram observações nessa faixa em quatro anos consecutivos. Dessas onze cooperativas de crédito, seis são de Santa Catarina, duas do Rio Grande do Sul, uma de São Paulo, uma de Minas Gerais e uma do Mato Grosso; além de que durante o período analisado sete delas apresentaram $100 \%$ de eficiência no mínimo em um dos anos analisados. É importante notar que esse grupo de eficiência mediana apresentou mais observações que gerenciaram seus resultados durante o período analisado do que observações que não gerenciaram, o que confirma o teste qui-quadrado de que há relação entre as variáveis analisadas.

\section{Considerações finais}

Esta pesquisa objetivou avaliar se existe relação entre o gerenciamento de resultados para evitar divulgar perdas e os escores de eficiência nas cooperativas de crédito filiadas ao sistema Unicred durante o período de 2009 a 2014. Para tanto, utilizou-se o teste não paramétrico de $\chi^{2}$ numa amostra de 90 observações. Os resultados do teste estatístico de $\chi^{2}$ encontrados na pesquisa, indicaram que há relação entre o gerenciamento de resultados para evitar divulgar perdas e os escores de eficiência nas cooperativas de crédito associadas à Unicred durante o período analisado. $\mathrm{O}$ que permitiu realizar inferências sobre as faixas de eficiência e a ocorrência ou não de gerenciamento de resultados.

Destaca-se, dentre os resultados, que a maioria das cooperativas de crédito que obtiveram $100 \%$ de eficiência durante o período analisado não gerenciou seus resultados, e a maioria das cooperativas dentro dessa faixa de eficiência encontra-se no Estado de Santa Catarina. Outro importante achado deste estudo refere-se ao fato de que a faixa de eficiência que mais gerenciou seus resultados a fim de evitar divulgar perdas foi a de eficiência na faixa mediana, com escores entre $60 \%$ a $80 \%$, permitindo inferir que as cooperativas mais eficientes não necessitariam gerenciar seus resultados e que as cooperativas menos eficientes não teriam nenhum tipo de benefício ao adotar tal prática. A maioria das cooperativas na faixa de eficiência mediana também localiza-se no estado de Santa Catarina, sendo que em pelo menos um dos anos do período analisado, estas alcançaram $100 \%$ de eficiência.

A pesquisa vê-se importante dentro da temática de cooperativismo de crédito, tema ainda pouco explorado na literatura brasileira. Adicionalmente, não se tem conhecimento de outro trabalho que abordou as variáveis apresentadas nesta pesquisa em conjunto, a saber, gerenciamento de resultados e eficiência nas cooperativas de crédito, o que agrega, portanto, informações relevantes para análises desse grupo de instituições financeiras.

Por fim, sugere-se para pesquisas posteriores, que seja realizada uma análise similar para outros sistemas de cooperativas de crédito do Brasil a fim de obter um panorama completo sobre a relação entre gerenciamento de resultados e eficiência dentro do cenário do cooperativismo brasileiro. 


\section{Referências}

ANCOSOL - Associação Nacional do Cooperativismo de Crédito da Economia Familiar e Solidária. Disponível em: <http://www.ancosol.org.br/cooperativismo.ph p>. Acesso em: 15 abr. 2015.

ANDERSON, D. R., SWEENEY, D. J., WILLIAMS, T. A. Estatística Aplicada à Administração e Economia. São Paulo, Pioneira Thomson Learning, 2003.

ARAÚJO, E. A. T.; SILVA, W. A. C. Cooperativas de crédito: a evolução dos principais sistemas brasileiros com um enfoque em indicadores econômico-financeiros.

Revista Contemporânea de Economia e Gestão CONTEXTUS, v. 9, n. 1, p. 117-126, jan./jun. 2011. Disponível em: <http://www.contextus.ufc.br/index.php/contex tus/article/view/145>. Acesso em: 29 set. 2015.

BANCO CENTRAL DO BRASIL, Balancetes. Disponível em: http://www4.bcb.gov.br/fis/cosif/balancetes.asp . Acesso em: 23 maio 2016 a.

BANCO CENTRAL DO BRASIL, Relação de instituições em funcionamento no país. Disponível em: http://www.bcb.gov.br/fis/info/instituicoes.asp. Acesso em: 23 maio 2016b.

BANCO CENTRAL DO BRASIL, Dados Selecionados de Entidades Supervisionadas. Disponível em: https://www3.bcb.gov.br/informes/relatorios?li ngua $=$ pt. Acesso em: 9 jun. $2016 \mathrm{c}$.

BEUREN, I. M. Como elaborar trabalhos monográficos em contabilidade - teoria e prática. $3^{\mathrm{a}}$. ed. São Paulo: Atlas, 2008.

BERGER, A. N.; HUMPHREY, D. B. Efficiency of financial institutions: international survey and directions for future research. European Journal of Operational Research, v. 98, p. 175-212, 1997. Disponível em: <http://d1c25a6gwz7q5e.cloudfront.net/papers/ 67.pdf>. Acesso em: 9 nov. 2015.

BITTENCOURT, W. R. Rentabilidade em Bancos Múltiplos e Cooperativas de Crédito brasileiros. 2015. 215p. Dissertação (Mestrado em Ciências Contábeis), UFMG, Minas Gerais, 2015.

BRASIL. Legislação Cooperativista e Resoluções do Conselho Nacional de Cooperativismo. Lei Federal $n^{0} 5.764$ de 16 de dezembro de 1971. Define a política nacional de cooperativismo, inclui o regime jurídico das sociedades cooperativas e dá outras providências. Brasília, 1971. Disponível em: <http://www.planalto.gov.br/ccivil_03/leis/L57 64.htm>. Acesso em: 5 jun. 2017.

BRASIL. Lei no 4.595 de 31 de Dezembro de 1964 . Dispõe sobre a Política e as Instituições Monetárias, Bancárias e Creditícias, cria o Conselho Monetário Nacional e dá outras providências. Brasília, 1964. Disponível em http://www.planalto.gov.br/ccivil_03/leis/L459 5.htm. Acesso em: 5 jun 2017.

BRASIL. Conselho Monetário Nacional. Resolução $n^{\circ} 4.434$ de 5 de Agosto de 2015. Dispõe sobre a constituição, a autorização para funcionamento, o funcionamento, as alterações estatutárias e o cancelamento de autorização para funcionamento das cooperativas de crédito e dá outras providências, Brasília, 2015. Disponível em: http://www.bcb.gov.br/pre/normativos/busca/d ownloadNormativo.asp?arquivo=/Lists/Normat ivos/Attachments/48507/Res_4434_v1_O.pdf. Acesso em: 18jul. 2017.

\section{BRASIL. Lei Complementar $\mathbf{n}^{\mathbf{0}} 130$ de 17 de Abril de 2009.}

Dispõe sobre o Sistema Nacional de Crédito Cooperativo e revoga dispositivos das Leis $\mathrm{n}^{\text {os }} 4.595$, de 31 de dezembro de 1964, e 5.764, de 16 de dezembro de 1971. Disponível em <http://www.planalto.gov.br/ccivil_03/leis/LC P/Lcp130.htm>. Acesso em: 5 jun. 2017. 
BRESSAN, V. G. F.; BRAGA, M. J.; BRESSAN, A. A.; RESENDE FILHO, M. DE A. Uma proposta de indicadores contábeis aplicados às cooperativas de crédito brasileiras. Curitiba. Revista de Contabilidade e Controladoria, v. 2, n. 4, set./dez., p. 58-80, 2010.

BRESSAN, V. G. F.; SANTOS, L. S.; BRESSAN, A. A. Gerenciamento de Resultados para evitar divulgar perdas: Um estudo das cooperativas de crédito filiadas a Confesol. In: 53 Congresso da SOBER, 53, 2015a, João Pessoa. Anais... João Pessoa: SOBER, 2015a. p. 1-20. Disponível em: http://icongresso.itarget.com.br/tra/arquivo s/ser.5/1/4506.pdf. Acesso em: 30 set. 2015.

BRESSAN, V. G. F.; BRESSAN, A. A.; SILVA JÚNIOR, J. M. Evitar Divulgar Perdas: Foi Uma Estratégia Utilizada na Última Década pelas Cooperativas de Crédito Filiadas ao Sicredi?. RGC - Revista de Gestão e organizações cooperativas, Santa Maria, RS, v.2, n.3, p. 2742, jan./jun. 2015b.

BURGSTAHLER, D.; DICHEV, I. Earnings management to avoid earnings decrease and losses. Journal of Accounting \& Economics, North-Holland, v.24, n.1, p.99-126, Dec. 1997.

CONSELHO FEDERAL DE CONTABILIDADE (CFC). Resolução n ${ }^{\circ}$ 920, de 19 de Dezembro de 2001. NBC T 10.8. Aspectos Contábeis Específicos de Sociedades Cooperativas. Diário Oficial da União, Brasília, DF, 9 jan. 2002.

CONSELHO FEDERAL DE CONTABILIDADE (CFC). Resolução $n^{\circ}$ 959, de 14 de Março de 2003. NBC T 10.21 IT 2. Demonstração de Sobras e Perdas. Diário Oficial da União, Brasília, DF, 22 abr.2003.

CUEVAS, C. E.; FISCHER, K. P. Cooperative financial institutions: issues in governance, regulation, and supervision. Washington: The World Bank, 2006.

DAMBROS, M. A.; LIMA, J. F.; FIGUEIREDO, A. MA. Sistema cooperativo de crédito SICREDI: um estudo da eficiência das cooperativas do
Paraná. Gestão e Regionalidade, v. 25, n. 74, mai./ago., 2009.

DANTAS, J. A; MEDEIROS, O. R.de; LUSTOSA, P. R. B. O Papel de variáveis econômicas e atributos da carteira na estimação das provisões discricionárias para perdas em operações de crédito nos bancos brasileiros. BBR BRAZILIAN BUSINESS REVIEW, Vitória, v.10, n.04, p. 69-95, out. /dez. 2013.

FERREIRA, M. A. M.; GONÇALVES, R. M. L.; BRAGA, M. J. Investigação do desempenho das cooperativas de crédito de Minas Gerais por meio da Análise Envoltória de Dados (DEA). Economia Aplicada, São Paulo, v. $11, \quad$ n. $3, \quad$ p. 425-445, jul./set. 2007. Disponível em: http://www.scielo.br/scielo.php?pid=S141380502007000300006\&script=sci_arttext Acesso em: 26 set. 2015.

FUJI, A. H. A.; CARVALHO, L. N. G. de. Earnings Management no contexto bancário brasileiro. In: CONGRESSO USP, 5., 2005, São Paulo. Anais... São Paulo: USP, 2005. Disponível em: http://www.congressousp.fipecafi.org/web/artig os52005/an_resumo.asp. Acesso: 20 jul.2017.

GOULART, A. M. C. Gerenciamento de resultados contábeis em instituições financeiras no Brasil. 219 p. Tese (Doutorado em Ciências Contábeis) - FEA, Universidade de São Paulo, SP, 2007.

GRECCO, Marta Cristina Pelucio. O Efeito da convergência brasileira às IFRS no gerenciamento de resultados das empresas abertas brasileiras não financeiras. BBR BRAZILIAN BUSINESS REVIEW, Vitória, v.10, n.04, p. 117-140, out. /dez. 2013.

GUERRA, C. M. A. Relação entre eficiência e gerenciamento de resultados para evitar divulgar perdas nas cooperativas de crédito filiadas à Unicred. 2016. 35p. Monografia (Trabalho de Conclusão de Curso) Universidade Federal de Minas Gerais, Belo Horizonte, 2016. 
MAIA. S. C.; BRESSAN, V. G. F.; LAMOUNIER, W. M.; BRAGA, M. J. Gerenciamento de resultados em cooperativas de crédito no Brasil. Brazilian Busnisess Review. Vitória, v.10, n.4, p,96-116, out.-dez, 2013.

\section{MARTINEZ, A. L. Gerenciamento dos resultados} contábeis: estudo empírico das companhias abertas brasileiras. 2001. 154 f. Tese (Doutorado em Ciências Contábeis) - FEA, Universidade de São Paulo, SP, 2001.

MARTINEZ, A. L. Gerenciamento de resultados no Brasil: um survey da literatura. BBR Brazilian Business Review, Vitória, v.10, n.04, p. 01-31, out. /dez. 2013.

OCB - Organização das Cooperativas Brasileiras. Disponível em: http://www.ocb.org.br/o-que-ecooperativismo. Acesso em: 16 jul. 2017.

PERLINGUEIRO, B. de C. L. Teoria das Escolhas Contábeis: Fair Value de Derivativos em Bancos no Brasil. 2009. 184 p. Dissertação (Mestrado em Ciências Contábeis), FEA-USP, São Paulo, 2009.

PORTAL DO COOPERATIVISMO DE CRÉDITO, O que é uma cooperativa de crédito? Disponível em: http://cooperativismodecredito.coop.br/coopera tivismo/o-que-e-uma-cooperativa-de-credito/ Acesso em: 26 set. 2015.

\section{PORTAL DO COOPERATIVISMO} FINANCEIRO, Os pioneiros de Rochdale. Disponível em: http://cooperativismodecredito.coop.br/coopera tivismo/historia-do-cooperativismo/os-

pioneiros-de-rochdale/. Acesso em: 15 jun. 2017.

\section{PORTAL DO COOPERATIVISMO} FINANCEIRO, Legislação em vigor. Disponível em: http://cooperativismodecredito.coop.br/legislac ao-e-gestao/legislacao-em-vigor/. Acesso em: 15 jun. 2017.
PORTAL DO COOPERATIVISMO FINANCEIRO, Dados consolidados dos sistemas cooperativos. Disponível em: http://cooperativismodecredito.coop.br/cenariomundial/cenario-brasileiro/dados-consolidadosdos-sistemas-cooperativos/. Acesso em: 15 jun. 2017.

PORTAL DO COOPERATIVISMO FINANCEIRO, Expressão mundial. Disponível em: http://cooperativismodecredito.coop.br/cenariomundial/expressao-mundial/. Acesso em: 15 jun. 2017.

PORT, M. PORTAL DO COOPERATIVISMO FINANCEIRO, Cooperativismo Financeiro manteve crescimento em 2016. Disponível em:

http://cooperativismodecredito.coop.br/2017/05 /cooperativismo-financeiro-mantevecrescimento-em-2016/. Acesso em: 4 set. 2017

REIS, E. M. D.; LAMOUNIER, W.M.; BRESSAN, V.G.F. Um estudo empírico do gerenciamento de resultados por meio de decisões operacionais nas empresas abertas brasileiras. Revista Contabilidade e Finanças (Online), v.26, n.69, p.247-260, 2015.

RODRIGUES, A.; PAULO, E.; CARVALHO, L. N. Gerenciamento de resultados por meio das transações entre companhias brasileiras interligadas. RAUSP. Revista de Administração, v. 42, p. 216-226. 2007.

SANTOS, L. S. Z. Gerenciamento de Resultados para evitar divulgar perdas foi uma estratégia nas cooperativas filiadas à Unicred?. 2015. 50p. Monografia (Trabalho de Conclusão de Curso) - Universidade Federal de Minas Gerais, Belo Horizonte, 2015.

SILVA JUNIOR, J. M.; BRESSAN, V. G. F.;BRESSAN, A.A. Gerenciamento de Resultados em Cooperativas no Brasil: Avaliação do Income Smoothing às Filiadas do SICREDI. Advances in Scientific and Applied Accounting, v. 9, p. 283-300, 2016. 
SOARES, M. M.; MELO SOBRINHO, A. D. de. Microfinanças: o papel do Banco Central do Brasil e a importância do cooperativismo de crédito. Brasília: BCB, 2008. Disponível em: https://www.bcb.gov.br/htms/public/microcredi to/livro_microfinan\%E7as_internet.pdf. Acesso em: 20 jul 2017.

TABAK, B. M.; KRAUSE, K.; PORTELLA, G. R. Eficiência bancária: o valor intrínseco na função de produção. Revista de Administração, São Paulo, v. 40, n. 4, p. 361-379, out./nov./dez. 2005. Disponível em: http://www.spell.org.br/documentos/ver/16862/ eficiencia-bancaria--o-valor-intrinseco-nafuncao-de-producao Acesso em: 9 nov. 2015.

UNICRED - Confederação Nacional das Cooperativas Centrais Unicred's - Disponível em: http://www.unicred.com.br/. Acesso em: 19 jul. 2017.

VILELA, D. L.; NAGANO, M. S.; MERLO, E. M. Aplicação da análise envoltória de dados em cooperativas de crédito rural. Curitiba. Revista de Administração Contemporânea, v. 11, n. Edição Especial, p. 99-120, 2007.

WOCCU - World Council of Credit Unions. 2015 Statistical Report. 2017. Disponível em: <http://www.woccu.org/publications/statreport >. Acesso em: 20 jul. 2017.

\section{ZENDERSKY, H. C. Gerenciamento de resultados} em instituições financeiras no Brasil - 2000 a 2004. 131f. Brasília. Dissertação (Mestrado em Ciências Contábeis) - Programa Multiinstitucional e Inter-Regional de PósGraduação em Ciências Contábeis da Universidade de Brasília, Brasília, 2005. 